\section{Chemical Structure and Physical Properties of Cyclic Olefin Copolymers (IUPAC Technical Report)}

\author{
Ju Young Shin, Ji Yong Park, Chenyang Liu, \\ Jiasong He, and Sung Chul Kim \\ Pure and Applied Chemistry \\ Vol. 77, No. 5, pp. 801-814 (2005)
}

The modification of general-purpose polyolefin materials in order to enable their use as engineering plastics is currently a relevant topic for industrial as well as academic research. Cyclic olefin copolymers comprise one of the new classes of polymers based on cyclic olefin monomers and ethene. Because of the bulky cyclic olefin units randomly or alternately attached to the polymer backbone, the copolymer becomes amorphous and shows the properties of high glass-transition temperature, $T_{\text {g }}$, optical clarity, low shrinkage, low moisture absorption, and low birefringence.

With these properties, the application of cyclic olefin copolymer has now been extended to production of plastic lenses and optical storage media, and is currently being produced by Mitsui Chemical Co., Ticona (formerly Hoechst Celanese), Japan Synthetic Rubber, and Zeon Chemicals Co.

There are several types of commercial cyclic olefin copolymers based on different types of cyclic monomers and polymerization methods. Cyclic olefin copolymers are produced by chain copolymerization of cyclic monomers such as 8,9,10-trinorborn-2-ene

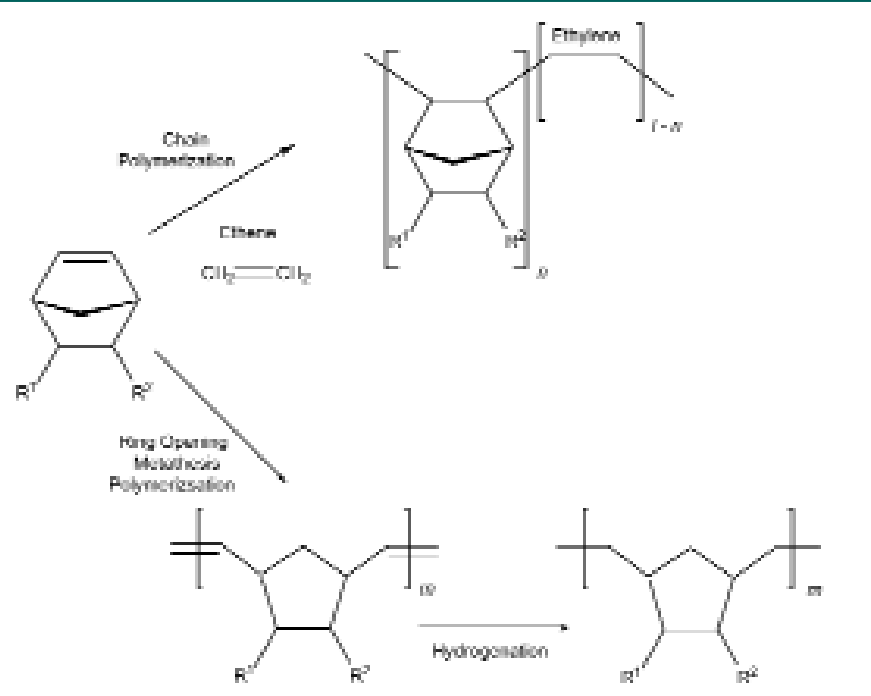

Typical polymerization scheme for a cyclic olefin polymer. (norbornene) or 1,2,3,4,4a,5,8,8a-octahydro-1,4:5,8dimethanonaphthalene (tetracyclododecene) with ethene, Ticona's TOPAS, Mitsui Chemical's APEL, or by ring-opening metathesis polymerization of various cyclic monomers followed by hydrogenation (Japan Synthetic Rubber's ARTON, Zeon Chemical's Zeonex and Zeonor).

Recently, a considerable amount of work has been reported on the physical properties and microstructure of cyclic olefin copolymers. Most of these reports correlated the composition, microstructure, and thermal properties of ethene-norbornene copolymer. A few studies have been made on the influence of chemical structure on the thermal properties of cyclic olefin copolymers. There is no report on the surface characteristics of these polymers.

This paper analyzes the chemical structure of the currently commercialized cyclic olefin copolymers by ${ }^{13} \mathrm{C} \mathrm{NMR}$, and investigates their glass-transition temperatures and surface characteristics. It was observed that the glass-transition temperature of cyclic olefin copolymers depended on the bulkiness of the main chain, and the number of rings had an important role in increasing the bulkiness of cyclic olefin copolymers. Cyclic olefin copolymers with polar substituents such as ester or ether groups showed high surface energy per area and peel strength.

www.iupac.org/publications/pac/2005/7705/7705x0801.html

\section{Polyaniline: Thin Films and Colloidal Dispersions (IUPAC Technical Report)}

\author{
Jaroslav Stejskal and Irina Sapurina \\ Pure and Applied Chemistry \\ Vol. 77, No. 5, pp. 815-826 (2005)
}

Polyaniline (PANI) is one of the most important and widely studied conducting polymers. It is easily prepared (e.g., by the oxidation of aniline with ammonium peroxydisulfate in acidic aqueous medium) and obtained as a precipitate. Such synthesis has recently been investigated within IUPAC project 1999-024-1400, "Polyaniline: Preparation of a Conducting Polymer," and a report has been published in PAC 74 857-867 (2002) 